\title{
Electrophysiological, Biochemical, and Bioinformatic Methods for Studying CFTR Channel
} Gating and its Regulation

László Csanády ${ }^{1}$, Paola Vergani ${ }^{2}$, Attila Gulyás-Kovács ${ }^{3}, \&$ David C. Gadsby ${ }^{3}$

${ }^{1}$ Department of Medical Biochemistry, Semmelweis University, Budapest, Hungary

${ }^{2}$ Department of Neuroscience, Physiology and Pharmacology, University College London, London, United Kingdom

${ }^{3}$ Laboratory of Cardiac/Membrane Physiology, The Rockefeller University, New York, USA

In "Cystic Fibrosis Diagnosis and Protocols, Volume I: Approaches to Study and Correct CFTR Defects." M.D. Amaral, and K. Kunzelmann, editors. Springer, New York. 443-469. 10.1007/978-1-61779-117-8_28.

\section{Correspondence to:}

David C. Gadsby

Laboratory of Cardiac/Membrane Physiology

Rockefeller University

1230 York Avenue

New York, NY 10065, USA

Tel: 212-327-8680

Fax: 212-327-7589

E-mail: gadsby@ rockefeller.edu 


\begin{abstract}
CFTR is the only member of the ABC (ATP binding cassette) protein superfamily known to function as an ion channel. Most other ABC proteins are ATP-driven transporters, in which a cycle of ATP binding and hydrolysis, at intracellular nucleotide binding domains (NBDs) powers uphill substrate translocation across the membrane. In CFTR, this same ATP-driven cycle opens and closes a transmembrane pore through which chloride ions flow rapidly down their electrochemical gradient. Detailed analysis of the pattern of gating of CFTR channels thus offers the opportunity to learn about mechanisms of function not only of CFTR channels, but also of their ABC transporter ancestors. In addition, CFTR channel gating is subject to complex regulation by kinase-mediated phosphorylation at multiple consensus sites in a cytoplasmic regulatory domain that is unique to CFTR. Here we offer a practical guide to extracting useful information about the mechanisms that control opening and closing of CFTR channels: on how to plan (including information obtained from analysis of multiple sequence alignments), carry out, and analyze electrophysiological and biochemical experiments, as well as on how to circumvent potential pitfalls.
\end{abstract}




\section{Introduction}

\subsection{Present understanding of regulation of CFTR channel gating by nucleotides}

CFTR's pair of cytoplasmic NBDs (NBD1, NBD2) are drawn together in head-to-tail orientation (1,2), as in all other ABC proteins, by two ATP molecules bound within composite interfacial catalytic sites, between the Walker A and B motifs of one NBD and the LSGGQ-like $\mathrm{ABC}$ signature sequence of the other $(\mathbf{3 , 4 , 5 , 6 , 7 , 8 , 9 )}$. Hydrolysis of the ATP disrupts the interface, causing the NBDs to separate and allowing fresh ATP to bind. In CFTR, these cyclic, ATPdriven motions of the NBDs are transmitted to the transmembrane domains (TMDs) to alternately open and close the pore through which chloride ions flow rapidly across the membrane, down their electrochemical gradient. Dissimilarities between NBD1 and NBD2 in CFTR, mirrored in all members of the $\mathrm{OAD}$ (organic anion and drug; 10) transporter subfamily of $\mathrm{ABC}$ proteins to which CFTR belongs, render only one of the composite interfacial ATP sites (the "NBD2 composite" site, incorporating the NBD2 Walker motifs) catalytically competent, while crippling the "NBD1 composite" site by substitutions on both sides of the interface. In the absence of ATP, phosphorylated (see 1.2., below) CFTR channels essentially remain closed (except for rare, brief, openings; e.g., 11,12). On exposure of the cytoplasmic surface of CFTR channels to millimolar ATP, its binding at the two interfacial sites causes NBD1-NBD2 heterodimerization and opening of the ion pore. The average channel opening rate is a saturable function of ATP concentration according to Michaelis Menten kinetics and is half maximal near $50 \mu \mathrm{M}$ ATP for wild-type (WT) CFTR (13). Because this apparent affinity for activation by ATP is diminished by mutations of either NBD that are expected to impair ATP binding, normal channel opening appears to require ATP binding in both composite sites (12).

The pore remains open until hydrolysis of the ATP in the competent "NBD2 site" triggers channel closure. That hydrolysis rate-limits closure is supported by prolongation of channel openings upon interference with hydrolysis by addition of inorganic phosphate analogues such as orthovanadate or pyrophosphate $(\mathbf{1 4 , 1 5 , 1 6 )}$, or of poorly hydrolyzable ATP analogs like AMPPNP $(17,15)$, or by mutation of key catalytic residues $(15,18,11,12,1)$. That the hydrolysis occurs only in the "NBD2 site", and not in the "NBD1 site", is demonstrated by the findings that

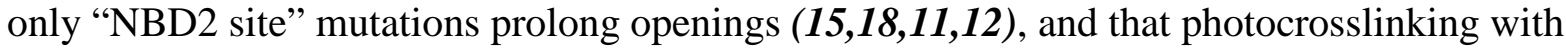

gamma- ${ }^{32} \mathrm{P}-8$ azidoATP radio-labels only the "NBD1 site", since the ${ }^{32} \mathrm{P}$ is released from the 
"NBD2 site" too rapidly to be observed $(\mathbf{1 9 , 2 0 )}$. The photocrosslinking experiments also indicated that non-hydrolyzed ATP remained bound for minutes at the "NBD1 site" $(\mathbf{1 9}, 20)$, arguing that the seconds-long ATP binding and hydrolysis cycle that drives CFTR channel gating reflects events at the "NBD2 site" $(\mathbf{1 5 , 1 2 , 2 0 )}$, a conclusion further supported by the much slower loss, after sudden ATP withdrawal, of the ability of brief exposures to pyrophosphate or AMPPNP to elicit prolonged channel openings (21).

Because crystal structures of nucleotide-bound $\mathrm{ABC}$ transporters show the extracellularside gate in the transmembrane domains open and the cytoplasmic side closed (e.g., 7,8,9,22), whereas both gates must be open in an ATP-bound CFTR channel to allow the observed rapid chloride ion flow (millions of ions per sec per channel), in CFTR the cytoplasmic-side gate would appear to have become atrophied, or uncoupled from the outer gate (23). CFTR can therefore be considered to be a broken $\mathrm{ABC}$ transporter (e.g., 24,25,26,27). Detailed analysis of the pattern of gating of CFTR channels thus offers the opportunity to learn about mechanisms of function not only of CFTR channels, but also of their ABC transporter ancestors.

\subsection{Present understanding of regulation of CFTR channel gating by phosphorylation}

Before a CFTR channel can be opened by ATP, cAMP-dependent protein kinase (PKA) must phosphorylate consensus-site serines in the $\sim 200$-residue cytoplasmic regulatory $(\mathrm{R})$ domain that links CFTR's two homologous halves $(28,29)$, and phosphorylation of PKC sites in the $\mathrm{R}$ domain might be a prerequisite $(\mathbf{3 0 , 3 1})$. Because cells contain millimolar levels of ATP, enough to saturate binding at CFTR's NBDs, phosphorylation of the R domain by PKA may be presumed to be the principal physiological regulator of CFTR channel activity. The R domain is unique to CFTR, and its structure and mechanism remain controversial and incompletely understood (reviewed in 32). Evidence of structural disorder of isolated $\mathrm{R}$ domain $(33,34)$, and of graded activation of CFTR channels with increasing phosphorylation (e.g., 28,35,36), have been interpreted as indicating redundancy among up to 9 R-domain phosphoserines, with accumulated negative charge as the major factor. The positions of consensus serine-containing segments in crystal structures of CFTR NBD1 extended by an R-domain fragment (37), and NMR measurements of NBD1-R domain interactions $(34,38)$, prompted the proposal that non-

phosphorylated R domain binds to the NBDs and interferes with ATP-mediated formation of the NBD1-NBD2 heterodimer, and hence with CFTR channel opening; phosphorylation of the R 
domain is proposed to release it from the NBDs, relieving the inhibition of gating. Such a simple mechanism seems unlikely, as neither serine-containing segment is needed for channels to close in the absence of ATP, regardless of phosphorylation status, and their deletion or mutation does not alter the strict dependence of channel gating on phosphorylation by PKA (39). Moreover, deletion of the entire NBD2 $(40,41)$ or introduction of "constitutive" mutations $(42)$, even the CF-causing mutation G551D (43), results in channels that gate regardless of the presence of ATP - but even this "spontaneous" activity remains strictly dependent on prior phosphorylation by PKA. Finally, two of the phosphoserines, including the one most readily generated by PKA $(29,36,44,45)$, seem to have an inhibitory, rather than stimulatory, influence on channel open probability $\left(\mathrm{P}_{\mathrm{o}}\right.$, see 3.4.2.) (46,36). A region of density interpreted as $\mathrm{R}$ domain that extends between NBDs and TMDs in a cryo-EM low-resolution structure of CFTR (47) seems more consistent with an alternative proposal, that the $\mathrm{R}$ domain somehow controls transmission of information from the NBDs to the channel gates (20). 


\section{Materials}

\subsection{Xenopus oocyte incubation media}

After injection with cRNA, oocytes are kept for $1-5$ days at $18{ }^{\circ} \mathrm{C}$ in solution containing (in mM): $83 \mathrm{NaCl}, 2 \mathrm{KCl}, 1 \mathrm{MgCl}_{2}, 1.8 \mathrm{CaCl}_{2}, 5 \mathrm{HEPES}$ (pH 7.5), plus $50 \mu \mathrm{g} / \mathrm{ml}$ Gentamycin $(48,13,12)$.

\subsection{Solutions used for electrophysiological recordings}

For recording in intact oocytes, the superfusion solution contains (in $\mathrm{mM}$ ): $83 \mathrm{NaCl}, 2$ $\mathrm{KCl}, 1 \mathrm{MgCl}_{2}, 5 \mathrm{HEPES}$ (pH 7.5). For excised patch recording, pipette solution contains (in $\mathrm{mM}$ ) $138 \mathrm{NMDG}-\mathrm{Cl}, 2 \mathrm{MgCl}_{2}, 5$ HEPES (pH 7.4), and bath solution contains (in mM) 138 NMDG-Cl, $2 \mathrm{MgCl}_{2}, 0.5$ EGTA, 5 HEPES (pH 7.1); for chloride-free solutions, sulphamate or gluconate replace chloride $(48,13,12)$. 


\section{Methods}

\subsection{Heterologous expression of human CFTR in various cell types including Xenopus oocytes}

\subsubsection{Expression plasmids}

For expression in Xenopus oocytes, CFTR mutants are generated from pGEMHE-CFTR (48) by mutation with Quikchange, verified by automated DNA sequencing, and in vitro transcribed using mMessage T7 polymerase to yield cRNA. For expression in HEK293T cells, pIRES-CFTR constructs are generated by subcloning the corresponding CFTR into the EcoRV site of the pIRES vector (20).

\subsubsection{Cell preparation}

Oocytes are isolated from anaesthethized adult female Xenopus laevis, and dispersed using collagenase, as described (13). HEK293T cells are maintained in DMEM medium supplemented with 10\% Fetal Bovine Serum, plated in 100-mm dishes and transfected with pIRES-CFTR (20)

\subsection{Biochemical analysis of CFTR regulation}

\subsubsection{Cysteine modification as a tool to study domain-domain interactions}

To supplement the minimal structural information available for CFTR and to directly examine changes in conformation associated with gating, sulphydryl-specific crosslinking can be used to probe proximity of pairs of target cysteines introduced into CFTR, provided that some or all of the 18 native Cys are first removed or shown not to interfere with the assay of target Cys reactivity. If the $\mathrm{C} \alpha$ atoms of residues approach each other to within 5-8 $\AA$, then target Cys at those positions should form disulphide bonds upon oxidation with copper phenanthroline. Bifunctional sulphydryl-specific reagents, e.g., based on maleimide (2) or methanethiosulphonate (MTS; 49,50), with variable-length linkers can provide upper estimates for the minimum separation of Cys pairs under the conditions of the assay. These reagents can be applied to functioning CFTR channels in vivo. For example, crosslinking of several target Cys pairs across CFTR's head-to-tail NBD1-NBD2 heterodimer interface by BMOE (bismaleimidoethane; reactive groups $\leq 8 \AA$ apart) in intact oocytes, assessed from large gels shifts of split CFTR channels with a target Cys in each half, has demonstrated that they must approach to within $8 \AA$ 
of each other at some point during the gating cycle (2). In inside-out membrane patches excised from oocytes, formation of a disulphide bond across the NBD1-NBD2 interface, between Cys at position 1248 in the NBD2 Walker A motif and 549 in the NBD1 LSGGQ motif, prevented closure of the crosslinked CFTR channels after withdrawal of ATP; but the channels closed when the disulphide bonds were reduced by dithiothreitol (2), further supporting the association of dimerized NBDs with open channels. Similarly, bifunctional MTS reagents were found, in gelshift assays, to crosslink NBD1 and NBD2 to cytoplasmic coupling helices that extend from the TMDs $(51,50)$ in a manner consistent with the "domain-swap" organization seen in the crystal structure of Sav1866 a prokaryotic multidrug transporter orthologue (7); when tested in insideout patches, in contrast to the open state stabilization caused by the NBD1-NBD2 crosslink (2), crosslinking either NBD to one of the coupling helices tended to impair channel opening $(\mathbf{5 1 , 5 0 )}$. These crosslinking experiments on CFTR have all used homology models to identify candidate target positions for Cys substitutions.

Modification of a single introduced target Cys, while assaying function as a monitor of reaction and its consequence, is an alternative way to probe CFTR channel structure and mechanism. Addition of N-ethylmaleimide (NEM) to the ATP-containing solution bathing inside-out patches irreversibly diminished the $\mathrm{P}_{\mathrm{o}}$ of CFTR channels with a single Cys introduced into Walker $\mathrm{A}$ or $\mathrm{ABC}$ signature motif of either composite interfacial site (52). Modification of the Walker A, but not the ABC signature, mutants became slower as ATP concentration was raised (52) and, at each [ATP], modification was much slower in the "NBD1" than in the "NBD2" composite site; these observations fit well with the present view of ATP-driven gating of CFTR channels (section 1.1, above).

Further structural and mechanistic information may be obtained by determining the gating-state dependence of modification of single Cys or of crosslinking of Cys pairs (e.g., 53).

\subsubsection{Studying the kinetics of CFTR phosphorylation}

Western blots of full-length (54) or split (2) CFTR, and Coomassie-stained gels or autoradiographs of isolated $\mathrm{R}$ domain peptide $(\mathbf{2 9 , 3 6 )}$, have all revealed mobility shifts induced by phosphorylation with PKA. By using low [ATP] to slow phosphorylation, taking samples at different times, and using SDS-PAGE followed by mass spectrometry of separated peptides, twodimensional phosphopeptide mapping, and/or point mutation to identify individual 
phosphoserines, the kinetics of phosphorylation of individual Ser can be assessed. With this approach, Ser768 was found to be the first one phosphorylated and phosphorylation of Ser737 was found to cause the major mobility shift (36). Kinetic analysis of several small synthetic peptides (10-24 residues), each encompassing one (or two) of the R-domain consensus PKA sites, showed that the peptide containing Ser768 was by far the preferred substrate for PKA (29), consistent with the results for isolated R domain and entire CFTR (36).

A difficulty in all biochemical studies of CFTR is availability of sufficient amounts of protein; expression and purification from Sf9 insect cells (55) and baby hamster kidney (BHK21) fibroblast cells (56) have proven most successful so far. The large amplification of electrophysiological assays allows circumvention of this difficulty, as some biochemical studies can be done in real time on CFTR channels in voltage-clamped excised membrane patches or whole cells. For example, phosphorylation by PKA catalytic subunit or by PKC, and dephosphorylation by specific phosphatases, of WT CFTR channels or mutants lacking one or more consensus phosphorylation sites, can be monitored by recording current in excised patches during direct application of exogenous kinases and/or phosphatases or inhibitors of endogenous phosphatases (e.g., 35,57,30,58,59,31,60,36). The presence of active endogenous phosphatases in excised patches even permits determination of PKA concentration dependence of CFTR channel activation, assayed as $\mathrm{P}_{\mathrm{o}}$; enhanced sensitivity to PKA in Ser768Ala mutants compared to WT CFTR (36) confirmed the inhibitory influence of phosphoserine 768 concluded from measurements of sensitivity to the phosphodiesterase inhibitor IBMX in intact oocytes (46). The enhanced sensitivity to phosphorylation resulted in substantial activation of Ser768Ala CFTR channels in resting oocytes, due to basal levels of PKA activity; phosphorylation of six Ser (including Ser768) in the R domains of WT CFTR channels in resting oocytes was confirmed by mass spectrometry (36).

\subsection{Electrophysiological recording of CFTR currents}

The possibility of following ion channel function using electrophysiological techniques make CFTR uniquely suited for basic structure/function studies among ABC transporters. However, some of its gating characteristics - e.g. relatively slow and non-equilibrium gating limit the usefulness of some techniques.

\subsubsection{Recording CFTR currents in intact cells by TEVC and whole-cell patch-clamp}


The two-microelectrode voltage-clamp (TEVC) method, and tight-seal current recording in the whole-cell configuration, allow little or no direct modification of the intracellular solution. However, as described above, the main factors regulating CFTR channel gating are phosphorylation and interaction with ATP, both acting on intracellular domains. In most intactcell studies CFTR is activated by extracellular application of membrane penetrating forskolin, which activates adenylate cyclase, and hence PKA, eventually resulting in an increase in whole cell conductance. For WT CFTR expressed in oocytes, held at $-50 \mathrm{mV}$, the inward current peaks $\sim 3$ minutes after forskolin addition. For CFTR mutants with low $\mathrm{P}_{\mathrm{o}}$, conductance can increase more slowly, e.g. reaching a 10-100 fold lower maximal conductance in 10-20 minutes. The very indirect control of the channel gates somewhat limits the kinetic information that can be obtained with these techniques, yet they remain useful for screening (e.g. 48,2), or studying extreme alterations of gating kinetics (e.g. 61). In addition, whole cell current recordings can be valuable for studying endogenous CFTR in its physiological environment, with only minimal experimental modification (methods described in 62).

\subsubsection{Recording CFTR currents in inside-out patches}

The experimenter has many more possibilities if, after sealing, the patch can be excised, so that the intracellular face of the patch is exposed. In Xenopus oocytes, this inside-out configuration is conveniently obtained by initially "cramming" the pipette towards the centre of the cell, before extricating it with the excised membrane patch. Because of the characteristic slow gating of CFTR channels, relatively simple perfusion systems (e.g. available from ALA Scientific Instruments, www.alascience.com), which allow complete exchange of the solution bathing the intracellular (outside) face of the patch within a fraction of a second, are sufficient for most experiments. Most of the channels present in the patch will be activated within a few minutes after switching to a solution in which catalytic subunit of PKA (130 units/ml) and MgATP (concentrations 2-5 mM) are present. PKA extracted from bovine heart is commercially available (Sigma cat \# P2645; note that recombinant PKA catalytic subunit, Promega cat \# V5161, was found to be much less active even using up to 300 units/ml). Millimolar [ATP] are saturating for most mutants, the WT apparent dissociation constant being $50 \mu \mathrm{M}$ (e.g. 13). Simple solutions with high $\mathrm{Cl}^{-}$, N-methyl-D-glucamine as a counterion (to minimize cationic conductances), lightly buffered, and containing EGTA and millimolar $\mathrm{Mg}^{2+}$ are used as bath 
solutions, while pipettes (bathing the extracellular face) are filled with a similar solution but lacking EGTA (e.g. 27, see 2.2, above).

In some expression systems the level of channel expression can be modulated. This can be easily obtained when using Xenopus oocytes, by altering amounts of cRNA injected (typically from $0.1 \mathrm{ng} /$ oocyte to $10 \mathrm{ng} /$ oocyte for WT CFTR), the time allowed for expression ( 12 hours to one week), the position of the patch with respect to the injection site (when injecting within the animal pole, patches close to the injection site show higher expression). To a lesser extent, when using mammalian cell lines, variation in expression levels - among different cell lines and/or among clones obtained from different transfections, - can be achieved (e.g. 63). The experimenter can thus obtain two different, complementary, sets of kinetic information: either monitoring individual channels during steady-state gating (3.3.2.1) or recording ensemble currents flowing through a high number of channels (3.3.2.2.).

\subsubsection{Recording individual CFTR channel currents in inside-out patches}

If the patch contains one or few (e.g. $\leq 8)$ channels the current "jumps" corresponding to the opening and closing of individual channel gates can be detected. Since the single-channel conductance of CFTR is small $(\sim 10 \mathrm{pS}, 64)$, the main practical problem in performing these experiments is keeping electrical noise as low as possible to improve the signal/noise ratio. Practical considerations on this subject are discussed at length in excellent texts $(\mathbf{6 5 , 6 6 )}$. Most important, the electrical seal resistance between glass pipette tip and cell membrane needs to be very large (with Xenopus oocytes, seals $>100 \mathrm{G} \Omega$ can be routinely obtained, using freshly stripped oocytes and $0.2 \mu \mathrm{m}$-filtered solutions) and line-frequency interference needs to be minimized (by carefully grounding conducting objects close to the amplifier headstage, avoiding ground loops, and shielding in a Faraday cage). In addition, several parameters of the experimental setup can be optimized to obtain low-noise records (67). In our labs, the patches are excised in a large fluid-filled "patch-pulling chamber", and then transferred to a "flow chamber" for recording (Fig. 1). Here, to minimize the capacitance of the immersed pipette tip, the patch is immersed in a thin film of solution flowing down the walls of the flow chamber, along the petri dish edge, and grounded (Figure 1). Luckily, CFTR's slow

gating characteristics are here an advantage, since the recording bandwidth need not be wide and 
filtering at 50-200 Hz does not remove events of interest for ATP- and phosphorylationdependent gating.

\subsubsection{Recording macroscopic, multi-channel, CFTR currents in inside-out}

patches

When studying low probability events or mutants whose gating is very slow, accumulation of sufficient events cannot be achieved within the patch lifetime by recording from one or a few channels. In these cases, information on channel gating regulation is most simply obtained by recording ensemble current flow across patches containing a large number (typically hundreds or thousands) of CFTR channels ("macropatches"). Recording from macropatches allows relative steady-state parameters, e.g., relative $\mathrm{P}_{\mathrm{o}}$ upon sudden change in $[\mathrm{ATP}](13)$, or relative $\mathrm{P}_{\mathrm{o}}$ in ATP vs. poorly-hydrolyzable analogues (12), to be easily obtained. Alternatively, kinetic information can be extracted from measurements of the time course of current change following a manoeuvre that affects CFTR gating, e.g. burst duration upon washout of ATP (see 3.5, below).

\subsection{Analysis of steady-state CFTR current recordings}

\subsubsection{Idealization of membrane currents}

The first step in the process of extracting information on channel gating kinetics from a raw current trace is to reconstruct the time series of channel opening/closing transitions. The output of this "idealization" procedure is an ordered list of events, each event described by a pair of numbers $l_{\mathrm{k}}, t_{\mathrm{k}} ; l_{\mathrm{k}}$ denotes the number of channels open during the $k$ th event and $t_{\mathrm{k}}$ its duration. This "events list" contains in a compact form all the information the raw data carry about gating.

The simplest idealization algorithm is the half-amplitude threshold crossing technique (68). In this procedure the baseline current and possible slow current drifts - not channel-related - are subtracted from the raw data. Ideal conductance levels are then set to integer multiples of the unitary current amplitude, and consecutive data points which fall within a half-amplitude distance of a given ideal conductance level are merged into an event. For reliable application of this simple procedure the signal-to-noise ratio must be sufficiently high to minimize halfamplitude threshold crossings caused by noise, while individual gating transitions should remain well resolved in time. In addition, the channels should open predominantly to a single, well 
defined, conductance level. These criteria are typically met for WT CFTR under standard conditions (e.g., $(69,18,11,13))$.

The half-amplitude procedure might present problems when using MOPS as an intracellular buffer (15), for certain pore mutants (70), or for murine CFTR channels, which open predominantly to subconductance levels (71). In such cases more elaborate algorithms are necessary for idealization. Time-course fitting (68) and hidden Markov modeling $(\mathbf{7 2 , 7 3 )}$ are procedures that allow for successful idealization of records with frequent subconductance openings and/or an insufficient signal-to-noise ratio; such algorithms are implemented by the free software packages HJCFIT (http://www.ucl.ac.uk/Pharmacology/dcpr95.html) and QuB (http://www.qub.buffalo.edu/), respectively.

\subsubsection{Kinetic analysis of single-channel current traces}

Channel open probability $\left(\mathrm{P}_{\mathrm{o}}\right)$, the fraction of time the pore is open, is simply obtained as the sum of all open-time durations in the events list divided by the total recording time. Although $\mathrm{P}_{\mathrm{o}}$ can be viewed as the physiologically relevant readout of gating in terms of chloride transport, it provides essentially no information on mechanism. For instance, mutations might simultaneously alter the rates of both gating transitions by up to 1000-fold with no large effect on $\mathrm{P}_{\mathrm{o}}$ (e.g., E1371Q, (1)), emphasizing the importance of kinetic analysis for mechanistic understanding.

WT CFTR channel gating is characterized by bursts of openings - groups of openings, interrupted by brief closures - that are flanked by long, interburst closures. Correspondingly, dwell-time analysis of single WT channels reveals a single open state and two distinct closed states (69). The 3-state schemes $\mathrm{C}_{1}-\mathrm{O}-\mathrm{C}_{2}$ and $\mathrm{C}_{1}-\mathrm{C}_{2}-\mathrm{O}$ can equally well account for such a pattern of gating; assuming either scheme, the respective four rate constants can be estimated by maximum likelihood fitting. Two commonly used approaches for maximum likelihood fitting of single-channel events lists are fitting the open- and closed-time distributions (e.g., (74)), or fitting the entire series of closed and open-time durations (75,76,77), implemented, e.g., by the MIL subprogram of the QuB package, and by HJCFIT). In theory, the latter approach is more powerful because it exploits the information carried by correlations between the durations of

adjacent closed and open events. However, for schemes with a single open state (or, in general, 
when a single "gateway" state connects the sets of open and closed states), like the two schemes above, open and closed events are uncorrelated and the two approaches therefore equivalent.

It is important to note that the schemes $\mathrm{C}_{1}-\mathrm{O}-\mathrm{C}_{2}$ and $\mathrm{C}_{1}-\mathrm{C}_{2}-\mathrm{O}$ cannot be distinguished in steady-state records. And, because of the short life time of state $\mathrm{C}_{2}\left(\sim 10 \mathrm{~ms}\right.$ at $\left.25^{\circ} \mathrm{C}\right)$ relative to $\mathrm{C}_{1}\left(\sim 1 \mathrm{~s}\right.$ at $\left.25^{\circ} \mathrm{C}\right)$, a distinction based on the time course of current change upon sudden addition/removal of the ligand ATP is technically not feasible. Thus, at present there is no strong evidence to support a choice between them. Correspondingly, both schemes have been used to interpret ATP-dependence of CFTR gating, and fitting either scheme suggests [ATP]-dependence of a single rate: rate $\mathrm{C}_{1} \rightarrow \mathrm{O}(\mathbf{1 2})$ and rate $\mathrm{C}_{1} \rightarrow \mathrm{C}_{2}$, respectively (69). Because these rates are meaningful only in the context of a particular scheme, it is customary to report only the mean durations of open bursts and interburst closures, which are model-independent descriptive parameters, that can be calculated from the fitted rate constants regardless of the chosen scheme. Using this descriptive nomenclature it is generally agreed that increasing ATP concentrations shorten the mean interburst duration in a saturable fashion, while the mean burst duration, the mean intraburst closed time, and the mean number of intraburst closures per burst are little influenced by ATP concentration $(78,13,12,79)$.

An alternative approach for studying the kinetics of open bursts and interburst closures is provided by conventional burst analysis. This method consists of ignoring brief intraburst closures by creating a new events list of bursts and interbursts in which closures shorter than a specified cutoff are suppressed. This cutoff is chosen based on the distribution of closedtime durations in the original events list, and is ideally much larger than the mean duration of intraburst closures, but much smaller than that of interburst closures. Nevertheless, because the durations of both types of closures are exponentially distributed and therefore overlap, some fraction of closures will be necessarily misassigned regardless of the choice of the cutoff. This means that some fraction of intraburst closures will be erroneously kept and treated as interburst, and some fraction of interburst closures will be erroneously misclassified as intraburst and so eliminated. Two commonly used strategies for calculating the cutoff duration are to equalize the probabilities of the two types of error (80) or to minimize the total probability of committing any error (81). The merit of the first strategy is that the mean duration of reconstructed bursts provides an undistorted estimate of the true mean burst duration, whereas the second strategy was 
found to cause less distortion of the shape of the distribution of burst durations for a nonequilibrium cyclic gating mechanism like that of CFTR (27).

Because ATP concentration affects bursting, but not intraburst, kinetics, restricting kinetic analysis to a mere extraction of mean burst and interburst durations is equivalent to modeling ATP dependent gating by a simple two-state process. Though more informative than just extracting $\mathrm{P}_{\mathrm{o}}$, such a simplifaction severely limits the amount of information that can be obtained about a complex process involving binding of ATP at two distinct sites, dynamic formation and disruption of an NBD1/NBD2 heterodimer, and hydrolysis of ATP in one of the composite binding sites. However, by reconstructing each burst, burst analysis offers the advantage of providing also the distributions of burst and interburst durations, not just their mean values. These distributions carry invaluable information on the mechanism of bursts, and allow quantitative evaluation of alternative gating schemes by fitting them to the observed distributions using maximum likelihood. Although as a first approximation both interburst and burst durations are reasonably fit by single-exponential distributions $(18,13,12)$, careful fitting of large sets of both interburst (11) and burst (27) durations have identified tell-tale deviations from singleexponential behaviour that are indisputable signs of a highly non-equilibrium mechanism. Thus, the clear rising phases of these distributions (see histogram of burst durations in Fig. 2B) signal violation of microscopic reversibility (68). In addition, fitting these distributions by a cyclic mechanism (Fig. 2A; cf., 1.1) allows estimation of the rates of conformational transitions that are not associated with pore opening or closure (27).

\subsubsection{Kinetic analysis of multi-channel current traces}

Because CFTR gating is a slow process and gradual dephosphorylation by membranebound phosphatases limits the length of steady-state single-channel recordings, obtaining sufficient numbers of gating events from a patch containing only a single channel can become challenging. In contrast, from patches with multiple (e.g., 2-8) active channels comparable numbers of gating events are obtained in proportionately shorter duration recordings. An ensemble of several $(N)$ channels gating via a common mechanism but independently of each other can be described by a single macro-system with $N+1$ conductance levels; transition rates between states of the macro-scheme are functions of the single-channel transition rates. Consequently, both maximum likelihood analysis approaches mentioned in 3.4.2. above, i.e., 
fitting of dwell-time distributions and fitting the entire time series, can be generalized to a multichannel system. However, because the macro-system has many states, the computational burden for the latter approach becomes prohibitively large; even with improved present day (2010) PC performances, fitting the entire time series for a record with more than 2-3 channels is still impractical. In contrast, maximum likelihood fitting of single-channel open- and closed-time distributions can be readily generalized to a simultaneous fitting of the ensemble of the dwelltime distributions of all conductance levels of a multichannel patch. The computational burden of this approach is far smaller, because once the sequence in which the events follow each other is disregarded, the individual dwell-time distributions can be binned into histograms, resulting in a large reduction in the size of the data set to be fitted. Moreover, there is no disadvantage to losing correlation information between neighboring events for the simple $\mathrm{C}_{1}-\mathrm{O}-\mathrm{C}_{2}$ and $\mathrm{C}_{1}-\mathrm{C}_{2}-\mathrm{O}$ schemes (as noted above, 3.4.2.). Finally, a first-order correction for missed events due to limited recording bandwidth is also readily incorporated into this approach (82). A program implementing these procedures has been used successfully by several groups and is freely available upon request (laszlo.csanady@eok.sote.hu).

\subsection{Analysis of macroscopic current relaxations}

Information on gating kinetics of single ion channels can also be obtained from the time courses of macroscopic current relaxations in response to step changes in ligand concentration or voltage. These time courses can be fitted with sums of exponentials, whose time constants and fractional amplitudes are functions of the single-channel gating parameters. Non-linear least squares fitting algorithms are included in most commercially available data acquisition software packages (e.g., Pclamp, PulseFit).

Because, for CFTR, closing from a burst is little sensitive to ATP concentration and the rate of opening to a burst in the absence of ATP is vanishingly small, the time constant of macroscopic current relaxation upon sudden removal of ATP reflects the steady-state mean burst duration $(83,13,27)$. This technique for estimating mean burst duration has been preferentially used for catalytic site mutants which abolish ATP hydrolysis at the composite NBD2 site, (e.g., K1250A) or when non-hydrolyzable ATP analogs (e.g., AMP-PNP, pyrophosphate) are applied, because in either case burst durations are prolonged to several seconds or tens of seconds. While 
this makes it difficult to collect sufficient numbers of steady state single-channel gating events, macroscopic ATP-removal experiments are easy to perform, and require only moderately rapid solution exchange (e.g., 13,12,84,85).

One limitation of this approach is that for patches excised from certain cell types removal of PKA catalytic subunit results in a relatively abrupt shortening of mean burst durations, likely due to rapid partial dephosphorylation of CFTR channels by membrane-bound phosphatases $(\mathbf{1 7}, 13)$. In such systems the above approach can be used to study the mean burst duration of partially, but not of fully, phosphorylated channels. This is because sudden removal of ATP terminates not only channel opening, but also activity of the co-applied PKA catalytic subunit, and so full phosphorylation of the channels throughout the time course of current relaxation cannot be guaranteed.

\subsection{Thermodynamic approaches to studying the energetics of gating}

\subsubsection{Studying temperature dependence}

Observing a chemical reaction at several different temperatures provides insight into the energetics of the process. For an equilibrium reaction $\mathrm{A} \leftrightarrow \mathrm{B}$ the standard enthalpy change $\Delta H^{\mathrm{o}}$ between states B and A can be calculated from the van't Hoff plot, which displays the natural logarithm of the equilibrium constant $\left(\ln K_{\text {eq }}\right)$ as a function of the inverse of absolute temperature $(1 / T)$. The slope of this linear plot is $-\Delta H^{\mathrm{o}} /(R T)$, where $R$ is the gas constant $\left(8.31 \mathrm{Jmol}^{-1} \mathrm{~K}^{-1}\right)$. If not only the equilibrium constant, but also the rates of transition between states A and B can be measured, then temperature dependence of these rates can be used to calculate the activation enthalpies $\left(\Delta H^{\star}\right)$, i.e., the standard enthalpy differences between the transition state and the stable ground states $\mathrm{A}$ and $\mathrm{B}$. With $k_{\mathrm{AB}}$ denoting transition rate $\mathrm{A} \rightarrow \mathrm{B}$, plots of $\ln k_{\mathrm{AB}}$ (Arrhenius plot) or $\ln \left(k_{\mathrm{AB}} / T\right)$ (Eyring plot) as a function of $1 / T$ yield linear graphs with slopes $-E_{\mathrm{a}} /(R \mathrm{~T})$ or $\Delta H^{\ddagger} /(R T)$, respectively. Here, $E_{\mathrm{a}}$ denotes the activation energy and $\Delta H^{\ddagger}$ the enthalpy difference between the transition state and ground state $\mathrm{A}$; the two are related by the equation $E_{\mathrm{a}}=\Delta H^{\ddagger}+R T$. The corresponding parameters for the reverse step $\mathrm{B} \rightarrow \mathrm{A}$ can be obtained analogously from Arrhenius or Eyring plots of the reverse rate $k_{\mathrm{BA}}(\mathbf{8 6})$.

Ion channels are especially well suited to such thermodynamic studies, because the average rates of opening $\left(k_{\mathrm{o}}\right)$ and closure $\left(k_{\mathrm{c}}\right)$ can be readily measured in single-channel recordings. If channel gating is an equilibrium process, then the open-closed equilibrium constant 
is given by $K_{\mathrm{eq}}=\mathrm{P}_{\mathrm{o}} /\left(1-\mathrm{P}_{\mathrm{o}}\right)$; because $\mathrm{P}_{\mathrm{o}}=k_{\mathrm{o}} /\left(k_{\mathrm{o}}+k_{\mathrm{c}}\right)$, an equivalent formulation is $K_{\mathrm{eq}}=k_{\mathrm{o}} / k_{\mathrm{c}}$. Thus, measuring $\mathrm{P}_{\mathrm{o}}$ of such a single channel at various temperatures allows construction of a van't Hoff plot (i.e., $\ln \left[\mathrm{P}_{\mathrm{o}} /\left(1-\mathrm{P}_{\mathrm{o}}\right)\right]$ versus $\left.1 / T\right)$ for estimation of the standard enthalpy change between the open and closed ground states. Similarly, measuring $k_{\mathrm{o}}$ and $k_{\mathrm{c}}$ at various temperatures allows estimation of the activation enthalpies for opening and closure by construction of Arrhenius or Eyring plots.

However, in the case of WT CFTR there is strong evidence that the gating involves irreversible steps and therefore follows a non-equilibrium cycle $(15,87,11,27)$, such that channels close from a burst predominantly through a pathway different from that by which they open (see 1.2.). This circumstance has important implications for the interpretion of temperature dependence of WT CFTR bursting kinetics. First, if the process is not at equilibrium, then the apparent equilibrium constant $K_{\text {app }}=\mathrm{P}_{\mathrm{o}} /\left(1-\mathrm{P}_{\mathrm{o}}\right)$ is not a true equilibrium constant. Thus, construction of a van't Hoff plot is meaningless, as the slope of a plot of $\ln K_{\text {app }}$ versus 1/T does not report $\Delta H^{0}$ open-closed. (Note, that if $\Delta H^{0}$ for the process closed $\rightarrow$ open $\rightarrow$ closed is non-zero, then the quantity $\Delta \mathrm{H}^{\mathrm{o}}$ open-closed cannot even be defined in general; statements must be limited to comparing an open state to either the preceding or the subsequent closed state (85).) It is also important to note, that a linear van't Hoff plot does not imply an equilibrium mechanism, as has been argued (88). Because the apparent equilibrium constant $K_{\text {app }}=\mathrm{P}_{\mathrm{o}} /\left(1-\mathrm{P}_{\mathrm{o}}\right)$ is always equal to $k_{\mathrm{o}} / k_{\mathrm{c}}$, the relationship $\ln K_{\mathrm{app}}=\ln k_{\mathrm{o}}-\ln k_{\mathrm{c}}$ also holds regardless of the mechanism. Therefore, if the Arrhenius (or Eyring) plots for opening and closure are linear - as observed for CFTR $(\mathbf{8 8 , 8 9 , 8 5 )}$ - then the plot of $\ln K_{\text {app }}$ versus $1 / T$ will also be linear, regardless of the mechanism. Similarly, caution must be applied to the interpretation of energetic barriers. If WT CFTR indeed exits the open burst state through a pathway which involves ATP hydrolysis, then $\Delta H^{\ddagger}$ for opening and closure characterize single sides of two distinct energy barriers, rather than the two sides of a single barrier. In this case the barrier height for the reversal of the opening step must be obtained from the temperature dependence of closing rate under conditions that prevent ATP hydrolysis (e.g., using NBD2 composite catalytic site mutations or non-hydrolyzable ATP analogs). Together the three $\Delta H^{\ddagger}$ values for opening, non-hydrolytic closure and normal hydrolytic closure then allow reconstruction of the $\Delta H^{0}$ profile for a partial gating cycle which involves transition from an ATP-bound closed state through a transition state to the open state, and then from the open state to a second, distinct, transition state for closure (85). 
By transition state theory (e.g., 86,90) the absolute value of a transition rate $k$, measured at a given temperature, provides at least an upper estimate of the activation free energy $\left(\Delta G^{\ddagger}\right)$ in the form $\Delta \mathrm{G}^{\ddagger} \leq R T \ln \left(k_{\mathrm{B}} T /(k h)\right)$, where $k_{\mathrm{B}}$ is Boltzmann's constant $\left(1.38 \cdot 10^{-23} \mathrm{~J} /{ }^{\circ} \mathrm{K}\right)$ and $h$ is Planck's constant $\left(6.63 \cdot 10^{-34} \mathrm{Js}\right)$. This upper estimate of $\Delta G^{\ddagger}\left(\Delta G_{\text {max }}^{\ddagger}\right)$ together with the estimate of $\Delta H^{\ddagger}$ yields a lower estimate of the activation entropy $\Delta S^{\ddagger}: \Delta S_{\min }^{\ddagger}=\left(\Delta H^{\ddagger}-\Delta G^{\ddagger}{ }_{\max }\right) / T$ (cf., (85)) which provides additional information about the mechanisms underlying CFTR channel gating.

\subsubsection{Mutant cycle analysis}

The thermodynamic mutant cycle formalism (90) can be used to detect energetic coupling between two amino acid positions in a protein. In a generalized double mutant cycle, the WT, two single site mutants and the double mutant form the vertices of a thermodynamic cycle. From patch recordings of CFTR channel currents, several kinetic parameters can be measured that can be used to characterize WT and single and double mutants in terms of the $\Delta \mathrm{G}$ between two states. If the two residues do not interact, the effects of mutating one site will not depend on whether that mutation was done in a WT or mutant (at the other site) background; i.e., mutationlinked changes in $\Delta \mathrm{G}$ on parallel sides of the cycle are equal $\left(\Delta \Delta \mathrm{G}_{\mathrm{int}}=0\right)$. Any difference $\left(\Delta \Delta \mathrm{G}_{\mathrm{int}}\right.$ $\neq 0$ ) signifies, and (to some extent) quantifies, energetic coupling between the two residues.

Effective dissociation constants for ATP activation of channel gating, opening rates, and equilibrium constant between closed and open states in a non-hydrolytic background [footnote: after reducing the cyclic gating scheme to a closed-to-open equilibrium by impairing hydrolysis, this equilibrium constant can be obtained from $\mathrm{P}_{\mathrm{o}}$, as $K_{e q}=\mathrm{P}_{\mathrm{o}} /\left(1-\mathrm{P}_{\mathrm{o}}\right)$ ], can all be used to estimate a mutation-induced change in free energy, in each case probing a different step of the gating cycle. Several energetic coupling values can therefore be determined for each pair of target sites, allowing inferences to be made about how coupling between two positions changes as the NBDs bind ATP, and the channel opens, and transits between open and closed states, i.e., as the channel progresses through its gating cycle (1).

Unfortunately, interpretation of data obtained using the mutant cycle formalism is rarely straightforward. A first complicating factor relates to the large statistical variability observed in CFTR gating measurements. Because coupling energies are obtained as sums (of mutation-linked changes in $\Delta \mathrm{G}$ on parallel sides of the cycle), the errors on the individual sets of 
measurements are summed too, resulting in coupling energies, $\Delta \Delta \mathrm{G}_{\text {int }}$, which are not significantly different from zero unless the mutation effects are large. A second problem arises from the oversimplifying assumptions required to reduce kinetic data to free energy differences between two states. Thus, it is likely that the "equilibrium constants" estimated from measurements reflect the steady state distribution of channels among more than two underlying states; and states with low occupancy in WT might become important in certain mutants with severe phenotypes. A third problem can arise from the quantitative interpretation of $\Delta \Delta \mathrm{G}_{\text {int }}$ as interaction energy in the WT when the target-site residues still form significant interactions in the single and double mutants (90). Such interactions can usually be avoided by using alanine substitutions. However, mutant cycles in which the substitutions form a novel interaction in the double mutant only result in a stronger deviation from simple additivity, and this might help reveal energetic coupling when the single mutation effects are small.

\subsubsection{REFER analysis}

Rate-equilibrium free energy relationship (REFER) analysis provides information on transition state structures and has been used to study the temporal sequence in which various regions of an ion channel protein move during a closed-open conformational transition. REFER theory uses transition state formalism, and was developed for equilibrium reactions. The method assumes that structural perturbations introduced into a given region of an ion channel protein affect the free energy of the transition state for opening in proportion to their effects on the free energy of the open ground state (both free energies measured relative to the closed ground state); i.e., $\delta \Delta G_{\text {transition-closed }}^{\mathrm{o}} \Phi \cdot \delta \Delta G_{\text {open-closed }}^{\mathrm{o}}(0 \leq \Phi \leq 1)$. Because the logarithm of the opening rate $\left(k_{\mathrm{o}}\right)$ and of the closed-open equilibrium constant $\left(K_{\text {eq }}\right)$ are identically sensitive to $\Delta G_{\text {transition-closed }}^{\mathrm{o}}$ and $\Delta G^{\mathrm{o}}{ }_{\text {open-closed, }}$, respectively, the slope of a plot of $\ln k_{\mathrm{o}}$ as a function of $\ln K_{\text {eq }}$ for a series of structural perturbations to a particular region of a protein (Brønsted or REFER plot) provides the proportionality coefficient $\Phi$. (Because $\delta \Delta G_{\text {transition-closed }}^{\mathrm{o}} \Phi \cdot \delta \Delta G_{\text {open-closed }}^{\mathrm{o}}$ implies $\delta \Delta G_{\text {transition- }}^{\mathrm{o}}$

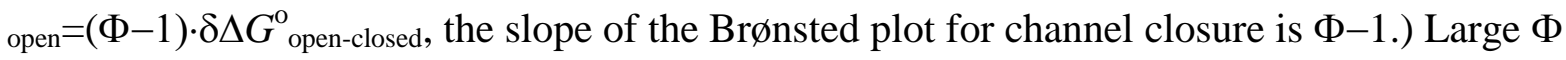
values (close to 1) indicate that in the transition state the perturbed region is already near its open-state conformation, while a $\Phi$ value close to 0 is an indication that the region is still closedlike in the transition state. Therefore, high $\Phi$ values are generally interpreted to signal early, and low $\Phi$ values late, movement of the target region during the closed-to-open transition (92). 
The success of REFER analysis for ligand-gated ion channels (e.g., 93,94) has encouraged its application to CFTR gating (95,96). Unfortunately, however, interpretation of REFER plots depends strictly on the assumption of an underlying equilibrium process. For this reason, although the observed linearity of the REFER plots and the complementarity of the slopes of such plots for opening and closure of CFTR channels have been argued to support an equilibrium mechanism (96), such inferences have no theoretical foundation. Indeed, it has been shown that both equilibrium and non-equilibrium mechanisms can result in either linear or nonlinear REFER plots, and that the complementary REFER slopes for opening and closure are a trivial feature of all ion channels regardless of their gating mechanism; moreover, for nonequilibrium mechanisms REFER analysis provides no information on the transition-state structures (97). These limitations should be considered before applying REFER analysis to CFTR.

\subsection{Bioinformatic approaches to identify coevolving amino-acid positions}

\subsubsection{Coevolution reports on functional residue-residue interactions}

Information about gating of CFTR channels can also be gleaned from the evolutionary record in the form of correlated amino acid substitutions. The conformational changes associated with gating are controlled by residue interactions, and interacting residues exert evolutionary pressure on each other, such that substitutions at one position are coupled to substitutions at another. In terms of multiple sequence alignments, this means that the variation of residues found in one column will correlate with the variation in some other column(s). This is illustrated schematically in Fig. 3 for evolution of positions $x$ and $y$ in a hypothetical protein family. In Fig. $3 \mathrm{~A}$, because of strong interaction between positions $x$ and $y$, their substitutions are correlated, leading to their coevolution, whereas in Fig. 3B the positions do not interact strongly, and so they evolve independently, and hence substitutions at those positions are uncorrelated $(\mathbf{9 8 , 9 9 ,} \boldsymbol{c f}$., 100).

Because CFTR belongs to the ABC protein superfamily, the large quantity of sequence data available makes such coevolution analysis attractive. Depending on which ABC subfamily sequences are included in the alignment, the analysis may illuminate general mechanisms shared by the whole $\mathrm{ABC}$ superfamily (e.g., interactions across the NBD dimer interface; 1) or may relate specifically to a particular subfamily. An alignment restricted to OAD 
family sequences, for instance, may be expected to yield information about mechanism applicable not only to CFTR but also to paralogs such as the SUR and MRP proteins.

\subsubsection{Statistical approaches for coevolution prediction}

In practice, given the protracted timescale of evolution, inferences about past substitution events made from alignments of contemporary sequences are used to generate a statistic that relates to the probability that two positions coevolved. A number of methods have been developed for these analyses, and they use one of two basic approaches (for recent reviews, see 98,101). In one, the coevolution statistic is derived from correlated patterns in the alignment; methods using this approach include McBASC (102), SCA (103), ELSC (104), OMES (105), CAPS (106), and MIp (107). In the other, an assumed evolutionary mechanism is used to reconstruct the history of amino-acid mutations, from which correlations in the substitution process can be extracted; this approach is used by CoMap $(108,109)$, BMM (110), CorrMut (111) and methods based on explicit coevolution models $(112,113)$. This latter approach adheres closely to the above (3.7.1.) definition of coevolution as temporally correlated substitutions, but is subject to the uncertainty inherent in inferring past substitution and branching events from present sequences.

\subsubsection{Input data and practical aspects}

A multiple sequence alignment comprises the input data for coevolution prediction. As misalignments increase with sequence divergence, filtering out distant sequences improves alignment quality but at the expense of discarding useful information. Calculation of BLAST Evalues (114) or HMMER log-odds likelihood scores (115), combined with estimates of local (116) or global (117) alignment quality, can help guide this tradeoff between sequence number

and divergence. Once sequences are aligned, gaps need to be removed by deleting entire columns and/or sequences. Ideally, the resulting multiple sequence alignment will retain hundreds of homologs each containing hundreds of positions.

\subsubsection{Validation of methods with structural contact prediction}

Benchmarking, to validate the predictive performance of a method on a test set of data under particular conditions, would ideally require a protein family for which all coevolving, and independently evolving, position pairs are known. As no such family exists, a data set can be simulated, which offers precise control over phylogenetic tree parameters and rates of evolution, and allows clear distinction between coevolving and independently evolving pairs. Applicability 
of the assumptions about mechanisms of (co)evolution used in the model, however, remains uncertain. An alternative approach is to use a known physical quantity, such as spatial proximity, to characterize position pairs. The ability of a method to predict coevolution that arose from structural contact can then be assessed for protein conformations for which a high-resolution 3D structure exists. In the absence of an X-ray crystal structure for CFTR, homology models (e.g., $\mathbf{5 1 , 1 1 8 , 1 1 9 )}$ based on the structure of $\operatorname{Sav1866}(\mathbf{7 , 8 )}$ provide a reasonable alternative $(\mathbf{1 1 9})$.

Coevolution analysis has been shown to predict structural contact between amino acids in proteins significantly $(120,109,121)$ better than random choice. Limits to the performance of coevolution analysis assessed by contact prediction include incomplete overlap between functional and structural interactions, in part due to functional interactions between spatially distant positions (connected by chains of direct, structural, interactions) that nevertheless induce coevolution (122). In general, correction for phylogenetic relatedness of sequences (123), instead of assuming sequence independence, improves performance $(124,125,126,110,107)$. In addition, coevolution analysis methods show different sensitivities to the heterogeneity of substitution rates across positions, i.e., conserved, slowly evolving positions versus rapidly evolving, variable positions $(127,128)$, and the accuracy of coevolution prediction may be improved by taking this into account.

In the case of CFTR, comparison of the results obtained from different methods of coevolution analysis, followed by structural contact tests, can exploit the strengths and overcome the weaknesses of individual methods, and so yield useful functional information. 


\section{References}

1. Vergani, P., Lockless, S.W., Nairn, A.C., and Gadsby, D.C. (2005) CFTR channel opening by ATP-driven tight dimerization of its nucleotide-binding domains. Nature 433, 876-880.

2. Mense, M., Vergani, P., White, D.M., Altberg, G., Nairn, A.C., and Gadsby, D.C. (2006) In vivo phosphorylation of CFTR promotes formation of a nucleotide-binding domain heterodimer. EMBO J. 25, 4728-4740.

3. Hopfner, K.P., Karcher, A., Shin, D.S., Craig, L., Arthur, L.M., Carney, J.P., and Tainer, J.A. (2000) Structural biology of Rad50 ATPase: ATP-driven conformational control in DNA double-strand break repair and the ABC-ATPase superfamily. Cell 101, 789-800.

4. Locher, K.P., Lee, A.T., and Rees, D.C. (2002) The E. coli BtuCD structure: a framework for ABC transporter architecture and mechanism. Science 296, 1038-1040.

5. Smith, P.C., Karpowich, N., Millen, L., Moody, J.E., Rosen, J., Thomas, P.J., and Hunt, J.F. (2002) ATP binding to the motor domain from an ABC transporter drives formation of a nucleotide sandwich dimer. Mol. Cell 10, 139-149.

6. Chen, T.Y., Chen, M.F., and Lin, C.W. (2003) Electrostatic control and chloride regulation of the fast gating of ClC-0 chloride. J. Gen. Physiol. 122, 641-651.

7. Dawson, R.J. and Locher, K.P. (2006) Structure of a bacterial multidrug ABC transporter. Nature 443, 180-185.

8. Dawson, R.J. and Locher, K.P. (2007) Structure of the multidrug ABC transporter Sav1866 from Staphylococcus aureus in complex with AMP-PNP. FEBS Lett. 581, 935-938.

9. Oldham, M.L., Khare, D., Quiocho, F.A., Davidson, A.L., and Chen, J. (2007) Crystal structure of a catalytic intermediate of the maltose transporter. Nature 450, 515-521.

10. Dassa, E.B.P. (2001) The ABC of ABCS: a phylogenetic and functional classification of ABC systems in living organisms. Research in Microbiology 152, 211-229.

11. Zeltwanger, S., Wang, F., Wang, G.T., Gillis, K.D., and Hwang, T.C. (1999) Gating of cystic fibrosis transmembrane conductance regulator chloride channels by adenosine triphosphate hydrolysis. Quantitative analysis of a cyclic gating scheme. J. Gen. Physiol. 113, $541-554$.

12. Vergani, P., Nairn, A.C., and Gadsby, D.C. (2003) On the mechanism of MgATP-dependent gating of CFTR Cl- channels. J. Gen. Physiol. 121, 17-36. 
13. Csanády, L., Chan, K.W., Seto-Young, D., Kopsco, D.C., Nairn, A.C., and Gadsby, D.C. (2000) Severed channels probe regulation of gating of cystic fibrosis transmembrane conductance regulator by its cytoplasmic domains. J. Gen. Physiol. 116, 477-500.

14. Baukrowitz, T., Hwang, T.C., Nairn, A.C., and Gadsby, D.C. (1994). Coupling of CFTR Clchannel gating to an ATP hydrolysis cycle. Neuron 12, 473-482.

15. Gunderson, K.L. and Kopito, R.R. (1995) Conformational states of CFTR associated with channel gating: the role ATP binding and hydrolysis. Cell 82, 231-239.

16. Carson, M.R., Winter, M.C., Travis, S.M., and Welsh, M.J. (1995b) Pyrophosphate stimulates wild-type and mutant cystic fibrosis transmembrane conductance regulator Cl- channels. J. Biol. Chem. 270, 20466-20472.

17. Hwang, T.C., Nagel, G., Nairn, A.C., and Gadsby, D.C. (1994) Regulation of the gating of cystic fibrosis transmembrane conductance regulator $\mathrm{C} 1$ channels by phosphorylation and ATP hydrolysis. Proc. Natl. Acad. Sci. U S A 91, 4698-4702.

18. Carson, M.R., Travis, S.M., and Welsh, M.J. (1995a) The two nucleotide-binding domains of cystic fibrosis transmembrane conductance regulator (CFTR) have distinct functions in controlling channel activity. J. Biol. Chem. 270, 1711-1717.

19. Aleksandrov, L., Aleksandrov, A.A., Chang, X.B. and Riordan, J.R. (2002) The First Nucleotide Binding Domain of Cystic Fibrosis Transmembrane Conductance Regulator Is a Site of Stable Nucleotide Interaction, whereas the Second Is a Site of Rapid Turnover. J. Biol. Chem. 277, 15419-15425.

20. Basso, C., Vergani, P., Nairn, A.C., and Gadsby, D.C. (2003) Prolonged nonhydrolytic interaction of nucleotide with CFTR's NH2-terminal nucleotide binding domain and its role in channel gating. J. Gen. Physiol. 122, 333-348.

21. Tsai, M.F., Shimizu, H., Sohma, Y., Li, M., and Hwang, T.C. (2009) State-dependent modulation of CFTR gating by pyrophosphate. J. Gen. Physiol. 133, 405-419.

22. Ward, A., Reyes, C.L., Yu, J., Roth, C.B., and Chang, G. (2007) Flexibility in the ABC transporter MsbA: Alternating access with a twist. Proc. Natl. Acad. Sci. U S A 104, 19005-19010.

23. Gadsby, D.C. (2009). Ion channels versus ion pumps: the principal difference, in principle. Nat. Rev. Mol. Cell. Biol. 10, 344-352. PMCID: PMC2742554. 
24. Jordan, I.K., Kota, K.C., Cui, G., Thompson, C.H., and McCarty, N.A. (2008) Evolutionary and functional divergence between the cystic fibrosis transmembrane conductance regulator and related ATP-binding cassette transporter. Proc. Natl. Acad. Sci. U S A 105, 18865-18870.

25. Chen, T.Y. and Hwang, T.C (2008) CLC-0 and CFTR: chloride channels evolved from transporters. Physiol. Rev. 88, 351-387.

26. Muallem, D. and Vergani, P. (2009) ATP hydrolysis-driven gating in cystic fibrosis transmembrane conductance regulator. Philos. Trans. R. Soc. Lond. B. Biol. Sci. 364, 247255.

27. Csanády, L., Vergani, P., and Gadsby, D.C. (2010) Strict coupling between CFTR's catalytic cycle and gating of its $\mathrm{Cl}$ - ion pore revealed by distributions of open channel burst durations. Proc. Natl. Acad. Sci. U S A 107, 1241-1246.

28. Cheng, S.H., Rich, D.P., Marshall, J., Gregory, R.J., Welsh, M.J., and Smith, A.E. (1991) Phosphorylation of the R domain by camp-dependent protein kinase regulates the CFTR chloride channel. Cell 66, 1027-1036.

29. Picciotto, M.R., Cohn, J.A., Bertuzzi, G., Greengard, P., and Nairn, A.C. (1992)

Phosphorylation of the cystic fibrosis transmembrane conductance. J. Bio. Chem. 267, 12742-12752.

30. Jia, Y., Mathews, C.J., and Hanrahan, J.W. (1997) Phosphorylation by protein kinase C is required for acute activation of cystic fibrosis transmembrane conductance regulator by protein kinase A. J. Biol. Chem. 272, 4978-84.

31. Chappe, V., Hinkson, D.A., Zhu, T., Chang, X.B., Riordan, J.R., and Hanrahan, J.W. (2003) Phosphorylation of protein kinase $\mathrm{C}$ sites in NBD1 and the R domain control CFTR channel activation by PKA. J. Physiol. 548, 39-52.

32. Gadsby, D.C., Vergani, P., and Csanady, L. (2006) The ABC protein turned chloride channel whose failure causes cystic fibrosis. Nature 440, 477-483.

33. Ostedgaard, L.S., Baldursson, O., Vermeer, D.W., Welsh, M.J., and Robertson, A.D. (2000) A functional $\mathrm{R}$ domain from cystic fibrosis transmembreane conductance regulator is predominantly unstructured in solution. Proc. Natl. Acad. Sci. U S A 97, 5657-5662. 
34. Baker, J.M., Hudson, R.P., Kanelis, V., Choy, W.H., Thibodeau, P.H., Thomas, P.J., and Forman-Kay, J.D. (2007) CFTR regulatory region interacts with NBD1 predominantly via multiple transient helices. Nat. Struct. Mol. Bio. 14, 738-745.

35. Chang, X.B., Tabcharani, J.A., Hou, Y.X., Jenson, T.J., Kartner, N., Alon, N., Hanrahan, J.W. and Riordan, J.R. (1993) Protein kinase A (PKA) still activates CFTR chloride channel after mutagenesis of all 10 PKA consensus phosphorylation sites. J. Biol. Chem. 268, 11304-11311.

36. Csanady, L., Seto-Young, D., Chan, K.W., Cenciarelli, C., Angel, B.B, Qin, J., McLachlin, D.T., Krutchinsky, A.N., Chait, B.T., Nairn, A.C., and Gadsby, D.C. (2005b) Preferential phosphorylation of R-domain serine 768 dampens activation of CFTR channels by PKA. J. Gen. Physiol. 125, 171-181.

37. Lewis, H.A., Buchanan, S.G., Burley, S.K., Conners, K., Dickey, M., Dorwart, M., Fowler, R., Gao, X., Guggino, W.B., Hendrickson, W.A., Hunt, J.F., Kearins, M.C., Lorimer, D., Maloney, P.C., Post, K.W., Rajashankar, K.R., Rutter, M.E., Sauder, J.M., Shriver, S., Thiodeau, P.H., Thomas, P.J., Zhang, M., Zhao, X., and Emtage, S. (2004) Structure of nucleotide-binding domain 1 of the cystic fibrosis transmembrane conductance regulator. EMBO J. 23, 282-293.

38. Kanelis, V., Hudson, R.P, Thibodeau, P.H., Thomas, P.J., and Forman-Kay, J.D. (2010) NMR evidence for differential phosphorylation-dependent interactions in WT an DeltaF508 CFTR. EMBO J. 29, 263-277. PMCID: PMC2808376.

39. Csanady, L., Chan, K.W., Nairn, A.C., and Gadsby, D.C. (2005a) Functional roles of non conserved structural segments in CFTR's NH2-terminal nucleotide binding domain. $J$. Gen. Physiol. 125, 43-55.

40. Zerhusen, B. and Ma, J. (1999) Function of the second nucleotide-binding fold in the CFTR chloride channel. FEBS Lett. 459, 177-185.

41. Chan, K.W., Csanády, L., Nairn, A.C., and Gadsby, D.C. (1999) Deletion analysis of CFTR channel R domain using severed molecules. Biophys. J. 76, A405.

42. Wang, W., Wu, J., Bernard, K., Li, G., Wang, G., Bevensee, M.O., et al. (2010) ATPindependent CFTR channel gating and allosteric modulation by phosphorylation. Proc. Natl. Acad. Sci. U S A 107, 3888-3893. 
43. Bompadre, S.G., Sohma, Y., Li, M., and Hwang, T.C. (2007) G551D and G1349D, two CFassociated mutations in the signature sequences of CFTR, exhibit distinct gating defects. J. Gen. Physiol. 129, 285-98.

44. King, J.D., Jr., Fitch, A.C., Lee, J.K., McCane, J.E., Mak, D.O., Foskett, J.K., and Hallows, K.R. (2009) AMP-activated protein kinase phosphorylation of the R domain inhibits PKA stimulation of CFTR. Am. J. Physiol. Cell Physiol. 297, C94-C101.

45. Kongsuphol, P., Cassidy, D., Hieke, B., Treharne, K.J., Schreiber, R., Mehta, A., and Kunzelmann, K. (2009) Mechanistic insight into control of CFTR by AMPK. J. Biol. Chem. 284, 5645-5653.

46. Wilkinson, D.J., Strong, T.V., Mansoura, M.K., Wood, D.L., Smith, S.S., Collins, F.S., and Dawson, D.C. (1997) CFTR activation: additive effects of stimulatory and inhibitory phosphorylation sites in the R domain. Am. J. Physiol. 273, L127-L133.

47. Zhang, L., Aleksandrov, L.A., Zhao, Z., Birtley, J.R., Riordan, J.R., and Ford, R.C. (2009) Architecture of the cystic fibrosis transmembrane conductance regulator protein and structural changes associated with phosphorylation and nucleotide binding. J. Struct. Biol. 167, 242-251.

48. Chan, K.W., Csanády, L., Seto-Young, D., Nairn, A.C. and Gadsby, D.C. (2000) Severed molecules functionally define the boundaries of the cystic fibrosis transmembrane conductance regulator's $\mathrm{NH}_{2}$-terminal nucleotide binding domain. J. Gen. Physiol.116, 163-180.

49. Loo, T.W. and Clarke, D.M (2001) Defining the drug-binding site in the human multidrug resistance P-glycoprotein using a methanethiosulfonate analog of verapamil, MTSverapamil. J. Biol. Chem. 276, 14972-14979.

50. He, L., Aleksandrov, A.A., Serohijos, A.W., Hegedus, T., Aleksandrov, L.A., Cui, L., Dokholyan, N.V., and Riordan, J.R. (2008) Multiple membrane-cytoplasmic domain contacts in the cystic fibrosis transmembrane conductance regulator (CFTR) mediate regulation of channel gating. J. Biol. Chem. 283, 26383-26390.

51. Serohijos, A.W., Hegedus, T., Aleksandrov, A.A., He, L., Cui, L., Dokholyan, N.V., and Riordan, J.R. (2008) Phenylalanine-508 mediates a cytoplasmic-membrane domain contact in the CFTR 3D structure crucial to assembly and channel function. Proc. Natl. Acad. Sci. U S A 105, 3256-3261. 
52. Cotten, J.F. and Welsh, M.J. (1998) Covalent modification of the nucleotide binding domains of cystic fibrosis transmembrane conductance regulator. J. Biol. Chem. 273, 3187331879.

53. Zhang, Z.-R., Song, B. and McCarty, N.A. (2005b) State-dependent Chemical Reactivity of an Engineered Cysteine Reveals Conformational Changes in the Outer Vestibule of the Cystic Fibrosis Transmembrane Conductance Regulator. J. Biol. Chem. 280, $41997-$ 42003.

54. Seibert, F.S., Chang, X.B., Aleksandrov, A.A., Clarke, D.M., Hanrahan, J.W., and Riordan, J.R. (1999). Influence of phosphorylation by protein kinase A on CFTR at the cell surface and endoplasmic reticulum. Biochim. Biophys. Acta. 1461, 275-283.

55. Ramjeesingh, M., Li, C., Garami, E., Huan, L.J., Hewryk, M., Wang, Y., Galley, K., and Bear, C.E. (1997) A novel procedure for the efficient purification of the cystic fibrosis transmembrane conductance regulator (CFTR). Biochem. J. 327, 17-21.

56. Aleksandrov, L., Mengos, A., Chang, X., Aleksandrov, A., and Riordan, J.R. (2001) Differential interactions of nucleotides at the nucleotide binding domains of the cystic fibrosis transmembrane conductance regulator. J. Biol. Chem. 276, 12918-12923.

57. Berger, H.A., Travis, S.M., and Welsh, M.J. (1993) Regulation of the cystic fibrosis transmembrane conductance regulator $\mathrm{Cl}$ - channel by specific protein kinases and protein phosphatases. J. Biol. Chem. 268, 2037-2047.

58. Travis, S.M., Berger, H.A., and Welsh, M.J. (1997) Protein phosphate 2C dephosphorylates and inactivates cystic fibrosis transmembrane conductance regulator. Proc. Natl. Acad. Sci. U S A 94, 11055-11060.

59. Luo, J., Pato, M.D., Riordan, J.R., and Hanrahan, J.W. (1998) Differential regulation of single CFTR channels by PP2C, PP2A, and other phosphatases. Am. J. Physiol. 274, C1397-C1410.

60. Chappe, V., Hinkson, D.A., Howell, L.D., Evagelidis, A., Liao, J., Chang, X.B., Riordan, J.R., and Hanrahan, J.W. (2004) Stimulatory and inhibitory protein kinase C consensus sequences regulate the cystic fibrosis transmembrane conductance regulator. Proc. Natl. Acad. Sci. U S A 101, 390-395. 
61. Wilkinson, D.J., Mansoura, M.K., Watson, P.Y., Smit, L.S., Collins, F.S., and Dawson, D.C. (1996). CFTR: the nucleotide binding folds regulate the accessibility and stability of the activated state. J. Gen. Physiol. 107, 103-119.

62. Sheppard, D. N., Gray, M.A., Gong, X., Sohma, Y., Kogan, I., Benos, D. J., et al. (2004) The patch-clamp and planar lipid bilayer techniques: powerful and versatile tools to investigate the CFTR Cl- channel. J. Cyst. Fibros. 3, 101-108.

63. Thomas, P. and Smart, T.G. (2005) HEK293 cell line: A vehicle for the expression of recombinant proteins. J. Pharmacol. Toxicol. Methods 51, 187-200.

64. Bear, C., Li, C., Kartner, N., Bridges, R., Jensen, T., Ramjeesingh, M., et al. (1992) Purification and functional reconstitution of the cystic fibrosis transmembrane conductance regulator (CFTR). Cell 68, 809-818.

65. Sakmann, B. and Neher, E., Eds. (1995). Single-channel recording. New York, NY, Plenum Press, 700.

66. Ashley, R. H., Ed. (1995) Ion Channels: a practical approach. Practical Approach Series. Oxford, Oxford University Press, 328.

67. Benndorf, K. (1995). Low-noise recording. In Single-channel recording. Sakmann, B. and Neher, E. Eds. New York, NY, Plenum Press, 129-145.

68. Colquhoun, D. and Sigworth, F.J. (1995) Fitting and statistical analysis of single-channel records. In Single channel recording. Sakmann, B. and Neher, E., editors. Plenum Press, New York.

69. Winter, M.C., Sheppard, D.N., Carson, M.R., and Welsh, M.J. (1994) Effect of ATP concentration on CFTR Cl- channels: a kinetic analysis of channel regulation. Biophys. J. 66, 1398-1403.

70. Zhang, Z.R., Cui, G., Liu, X., Song, B., Dawson, D.C., and McCarty, N.A. (2005a) Determination of the functional unit of the cystic fibrosis transmembrane conductance regulator chloride channel. One polypeptide forms one pore. J. Biol. Chem. 280, 458-468.

71. Lansdell, K.A., Kidd, J.F., Delaney, S.J, Wainwright, B.J., and Sheppard, D.N. (1998) Regulation of murine cystic fibrosis transmembrane conductance regulator Cl- channels expressed in Chinese hamster ovary cells. J. Physiol. 512 (Pt 3), 751-764.

72. Venkataramanan, L. and Sigworth, F.J. (2002) Applying hidden Markov models to the analysis of single ion channel activity. Biophys. J. 82, 1930-1942. 
73. Qin, F. (2004) Restoration of single-channel currents using the segmental k-means method based on hidden Markov modeling. Biophys. J. 86, 1488-1501.

74. Sigworth, F.J. and Sine, S.M. (1987) Data transformations for improved display and fitting of single-channel dwell time histograms. Biophys. J. 52, 1047-1054.

75. Horn, R. and Lange, K. (1983) Estimating kinetic constants from single channel data. Biophys. J. 43, 207-223.

76. Ball, F.G. and Sansom, M.S. (1989) Ion-channel gating mechanisms: model identification and parameter estimation from single channel recordings. Proc. R. Soc. Lond. B. Biol. Sci. 236, 385-416.

77. Qin, F., Auerbach, A., and Sachs, F. (1996) Estimating single-channel kinetic parameters from idealized patch-clamp data containing missed events. Biophys. J. 70, 264-280.

78. Winter, M.C. and Welsh, M.J. (1997) Stimulation of CFTR activity by its phosphorylated R domain. Nature 389, 294-296.

79. Bompadre, S.G., Ai, T., Cho, J.H., Wang, X., Sohma, Y., Li, M., and Hwang, T.C. (2005a) CFTR gating I: Characterization of the ATP-dependent gating of a phosphorylationindependent CFTR channel (DeltaR-CFTR). J. Gen. Physiol. 125, 361-375.

80. Magleby, K.L. and Pallotta, B.S. (1983) Burst kinetics of single calcium-activated potassium channels in cultured rat muscle. J. Physiol. 344, 605-623.

81. Jackson, M.B., Wong, B.S., Morris, C.E., Lecar, H., and Christian, C.N. (1983) Successive openings of the same acetylcholine receptor channel are correlated in open time. Biophys. J. 42, 109-114.

82. Csanády, L. (2000) Rapid kinetic analysis of multichannel records by a simultaneous fit to all dwell-time histograms. Biophys. J. 78, 785-799.

83. Weinreich, F., Riordan, J.R., and Nagel, G. (1999) Dual effects of ADP and adenylylimidodiphosphate on CFTR channel kinetics show binding to two different nucleotide binding sites. J. Gen. Physiol. 114, 55-70.

84. Bompadre, S.G., Cho, J.H., Wang, X., Zou, X., Sohma, Y., Li, M., and Hwang, T.C., (2005b) CFTR gating II: Effects of nucleotide binding on the stability of open states. J. Gen. Physiol. 125, 377-394. 
85. Csanády, L., Nairn, A.C., and Gadsby, D.C. (2006) Thermodynamics of CFTR channel gating: a spr-eading conformational change initiates an irreversible gating cycle. J. Gen. Physiol. 128, 523-533.

86. Segel, I.H. (1993) Enzyme Kinetics. Behavior and Analysis of Rapid Equilibrium and Steady-State Enzyme Systems. John Wiley \& Sons Inc., New York.

87. Ishihara, H. and Welsh, M.J. (1997) Block by MOPS reveals a conformation change in the CFTR pore produced by ATP hydrolysis. Am. J. Physiol. 273, C1278-C1289.

88. Aleksandrov, A.A. and Riordan, J.R. (1998) Regulation of CFTR ion channel gating by MgATP. FEBS Lett. 431, 97-101.

89. Mathews, C.J., Tabcharani, J.A., and Hanrahan, J.W. (1998) The CFTR chloride channel: nucleotide interactions and temperature-dependent gating. J. Membr. Biol. 163, 55-66.

90. Fersht, A. (2002) Structure and Mechanism in protein science. 4 ed. W.H. Freeman and Company, New York.

91. Faiman, G. A. and Horovitz, A. (1996) On the choice of reference mutant states in the application of the double-mutant cycle method. Protein Eng. 9, 315-316.

92. Auerbach, A. (2007) How to turn the reaction coordinate into time. J. Gen. Physiol. 130, 543546.

93. Chakrapani, S., Bailey, T.D., and Auerbach, A. (2004) Gating dynamics of the acetylcholine receptor extracellular domain. J. Gen. Physiol. 123, 341-356.

94. Purohit, P., Mitra, A., and Auerbach, A. (2007) A stepwise mechanism for acetylcholine receptor channel gating. Nature 446, 930-933.

95. Scott-Ward, T.S., Cai, Z., Dawson, E.S., Doherty, A., Da Paula, A.C., Davidson, H., Porteous, D.J., Wainwright, B.J., Amaral, M.D., Sheppard, D.N., and Boyd, A.C. (2007) Chimeric constructs endow the human CFTR Cl- channel with the gating behavior of murine CFTR. Proc. Natl. Acad. Sci. U S A 104, 16365-16370.

96. Aleksandrov, A.A., Cui, L., and Riordan, J.R. (2009) Relationship between nucleotide binding and ion channel gating in cystic fibrosis transmembrane conductance regulator. $J$. Physiol. 587, 2875-2886.

97. Csanády, L. (2009) Application of rate-equilibrium free energy relationship analysis to nonequilibrium ion channel gating mechanisms. J. Gen. Physiol. 134, 129-136. 
98. Galtier, N., and Dutheil, J. (2007) Coevolution within and between genes. Genome Dyn. 3, 112.

99. Fitch, W.M. and Markowitz, E. (1970) An improved method for determining codon variability in a gene and its application to the rate of fixation of mutations in evolution. Biochem. Gene.t 4, 579-593.

100. Gutell, R.R., Larsen, N., and Woese, C.R. (1994) Lessons from an evolving rRNA: 16S and 23S rRNA structures from a comparative perspectice. Micro. Biol. 58, 10-26.

101. Codoñer, F.M., O'Dea, S., and Fares, M.A. (2008) Reducing the false positive rate in the non-parametric analysis of molecular coevolution. BMC Evol. Biol. 8, 106.

102. Olmea, O., and Valencia, A. (1997) Improving contact predictions by the combination of correlated mutations and other sources of sequence information. Fold Des. 2, S25-S32.

103. Lockless, S.W., and Ranganathan, R. (1999) Evolutionarily conserved pathways of energetic connectivity in protein families. Science 286, 295-299.

104. Dekker, J.P., Fodor, A., Aldrich, R.W., and Yellen, G. (2004) A perturbation-based method for calculating explicit likelihood of evolutionary co-variance in multiple sequence alignments. Bioinformatics 20, 1565-1572.

105. Kass, I., and Horovitz, A. (2002) Mapping pathways of allosteric communication in GroEL by analysis of correlated mutations. Proteins 48, 611-617.

106. Fares, M.A., and Travers, S.A.A. (2006) A novel method for detecting intramolecular coevolution: adding a further dimension to selective constraints analyses. Genetics 173, 923.

107. Dunn, S.D., Wahl, L.M., and Gloor, G.B. (2008) Mutual information without the influence of phylogeny or entropy dramatically improves residue contact prediction. Bioinformatics 24, 333-340.

108. Dutheil, J., Pupko, T., Jean-Marie, A., and Galtier, N. (2005) A model-based approach for detecting coevolving positions in a molecule. Mol. Biol. Evol. 22, 1919-1928.

109. Dutheil, J., and Galtier, N. (2007) Detecting groups of coevolving positions in a molecule: a clustering approach. BMC Evol. Biol. 7, 242.

110. Dimmic, M.W., Hubisz, M.J., Bustamante, C.D., and Nielsen, R. (2005) Detecting coevolving amino acid sites using Bayesian mutational mapping. Bioinformatics 21, Suppl 1:i126-135. 
111. Fleishman, S.J., Yifrach, O., and Ben-Tal, N.. (2004) An evolutionarily conserved network of amino acids mediates gating in voltage-dependent potassium channels. J. Mol. Biol. 340, 307-318.

112. Pollock, D.D., Taylor, W.R., and Goldman, N. (1999) Coevolving protein residues: maximum likelihood identification and relationship to structure. J. Mol. Biol. 287, 187198.

113. Yeang, C.-H., and Haussler, D. (2007) Detecting coevolution in and among protein domains. PLoS Comput. Biol. 3, e211.

114. Altschul, S.F., Gish, W., Miller, W., Myers, E.W., and Lipman, D.J. (1990) Basic local alignment search tool. J. Mol. Biol. 215, 403-410.

115. Eddy, S.R. (2009) A new generation of homology search tools based on probabilistic inference. Genome Inform. 23, 205-211.

116. Notredame, C., and Abergel, C. (2003) Using multiple alignment methods to assess the quality of genomic data analysis. Bioinformatics and Genomes: Current Perspectives. Horizon Scientific Press, Wymondham, UK. 30--55.

117. Lassmann, T., and Sonnhammer, E.L.L. (2005) Automatic assessment of alignment quality. Nucleic Acids Res. 33, 7120-7128. doi:10.1093/nar/gki1020.

118. Mornon, J.P., Lehn, P., and Callebaut, I. (2009) Molecular models of the open and closed states of the whole human CFTR protein. Cell. Mol. Life Sci. 66, 3469-3486.

119. Alexander, C., Ivetac, A., Liu, X., Norimatsu, Y., Serrano, J.R., Landstrom, A., Sansom, M., and Dawson, D.C. (2009) Cystic fibrosis transmembrane conductance regulator: using differential reactivity toward channel-permeant and channel-impermeant thiolreacgtive probes to test a molecular model for the pore. Biochemistry 48, 10078-10088.

120. Fodor, A.A., and Aldrich, R.W. (2004b) On evolutionary conservation of thermodynamic coupling in proteins. J. Biol. Chem. 279, 19046-19050.

121. Fuchs, A., Martin-Galiano, A.J., Kalman, M., Fleishman, S., Ben-Tal, N., and Frishman, D. (2007) Co-evolving residues in membrane proteins. Bioinformatics 23, 3312-3319.

122. Burger, L. and van Nimwegen, E. (2010) Disentangling direct from indirect co-evolution of residues in protein alignments. PLoS Comput. Biol. 6, e1000633.

123. Felsenstein, J. (1985) Phylogenies and the comparative method. American Naturalist 125, 1. 
124. Wollenberg, K.R., and Atchley, W.R. (2000) Separation of phylogenetic and functional associations in biological sequences by using the parametric bootstrap. Proc. Nat.l Acad. Sci. U S A 97, 3288-3291.

125. Tillier, E.R.M., and Lui, T.W.H. (2003) Using multiple interdependency to separate functional from phylogenetic correlations in protein alignments. Bioinformatics 19, 750755.

126. Noivirt, O., Eisenstein, M., and Horovitz, A. (2005) Detection and reduction of evolutionary noise in correlated mutation analysis. Protein Eng. Des. Sel. 18, 247-253.

127. Fodor, A.A., and Aldrich, R.W. (2004a) Influence of conservation on calculations of amino acid covariance in multiple sequence alignments. Proteins 56, 211-221.

128. Martin, L.C., Gloor, G.B., Dunn, S.D., and Wahl, L.M. (2005) Using information theory to search for co-evolving residues in proteins. Bioinformatics 21, 4116-4124. 


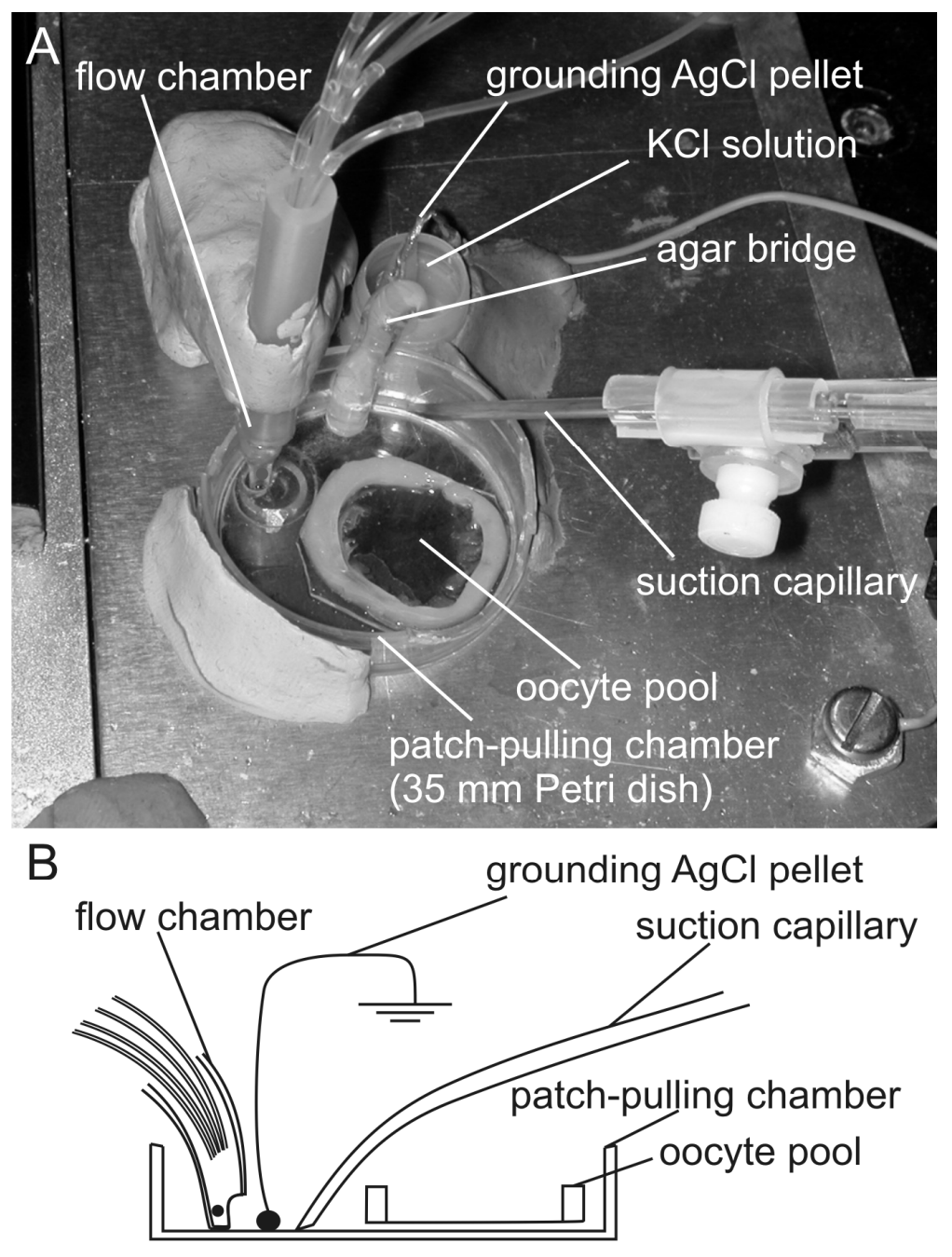

Figure 1. Photograph (A) and diagram (B) of a chamber for patch recording from oocytes.

The patch-pulling chamber (35 $\mathrm{mm}$ petri dish) and oocyte pool are initially filled with bath solution. An oocyte stripped of its vitelline membrane is placed in the oocyte pool, which can be rotated to position it appropriately for pipette approach. After the patch is excised it is transferred to the flow chamber (to the position indicated by the asterisk) by moving the microscope stage, the perfusion flow is turned on, and most of the bath solution is removed using the suction capillary. The oocyte in the oocyte pool remains submerged by solution, and can be used to provide further patches. The agar bridge (filled with $100 \mathrm{mM} \mathrm{KCl}+4 \%$ agarose, providing an electrical connection to a small reservoir filled with $\mathrm{KCl}$ ) is needed only when the bath solution does not contain $\mathrm{Cl}^{-}$, and is omitted from the diagram. 


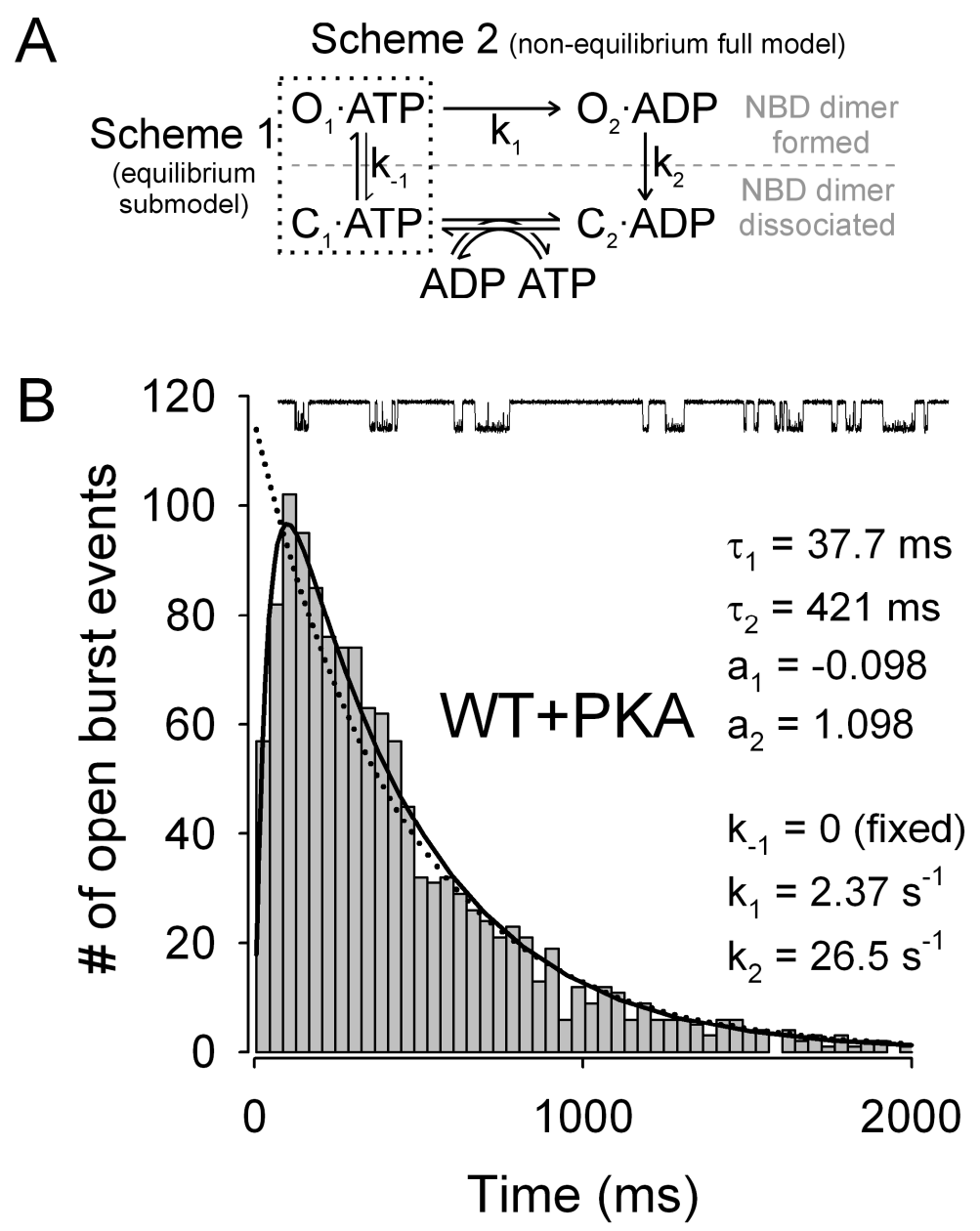

Figure 2. Distribution of open burst durations reports on mechanism of bursts. A, Cyclic scheme (Scheme 2) illustrating the proposed mechanism of CFTR gating in saturating ATP (see 1.1.). The nucleotide specified for each state represents that bound at the "NBD2-site"; the "NBD1-site" is assumed to remain ATP-bound in all four depicted states. Scheme 1, the subset of Scheme 2 framed by the dotted line, is a simple equilibrium closed-open model. Note that extraction of just mean burst and interburst durations - using either the $\mathrm{C}_{1}-\mathrm{O}-\mathrm{C}_{2}$ or $\mathrm{C}_{1}-\mathrm{C}_{2}-\mathrm{O}$ scheme - is equivalent to simplifying the mechanism of bursts to Scheme 1. B, Histogram of open burst durations, and 30-s segment of single-channel current recording, of WT CFTR gating in the presence of $2 \mathrm{mM}$ ATP $+300 \mathrm{nM}$ PKA. The dotted and solid lines are fits to Schemes 1 and 2, respectively; the fit parameters for Scheme 2, with predicted time constants and fractional amplitudes, are printed in the panel. The fit to Scheme 2 is ranked significantly $\left(\mathrm{P}=4 \cdot 10^{-9}\right)$ better by the log-likelihood ratio test. (Modified from 27.) 

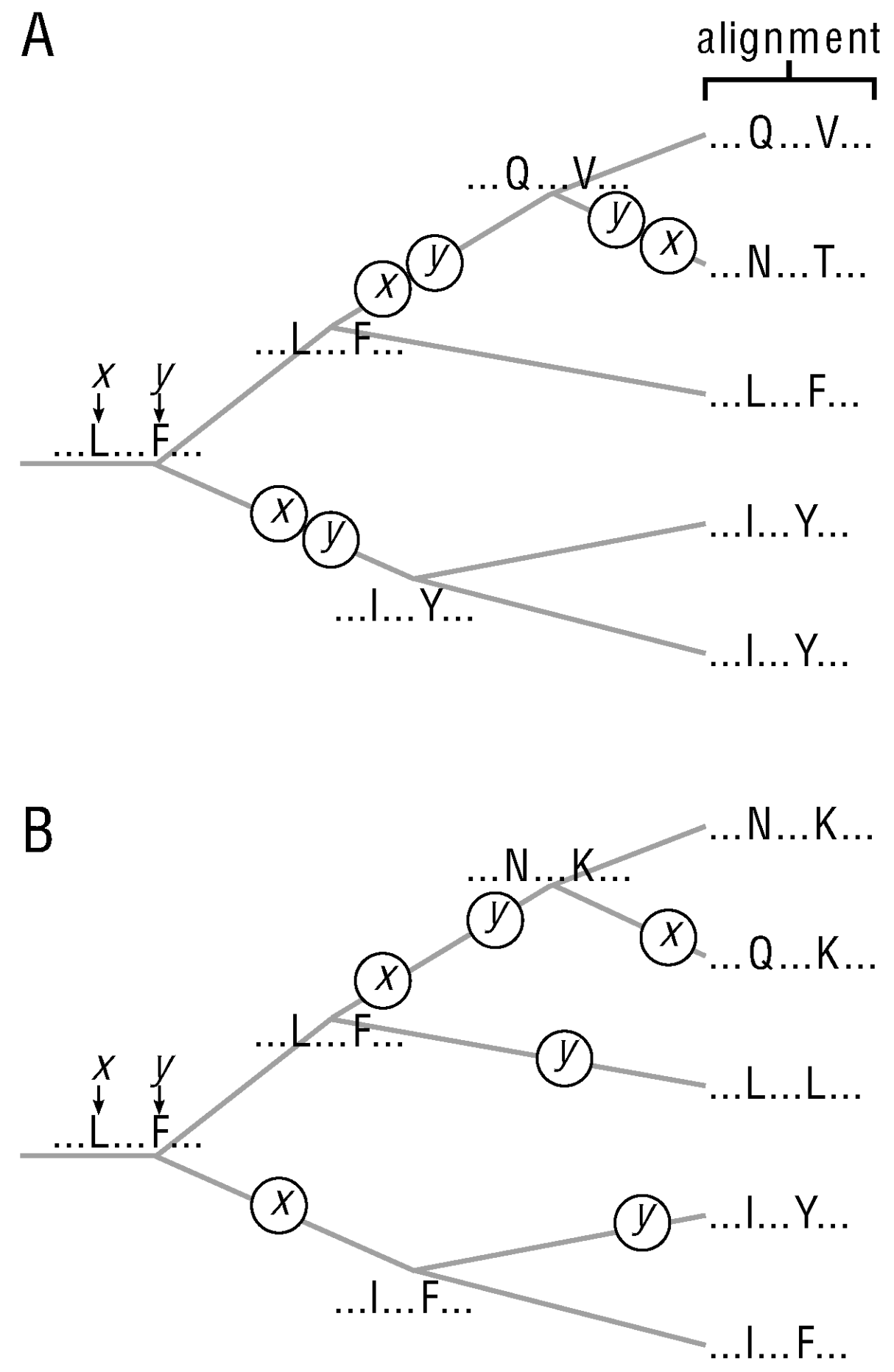
Figure 3. Signals of coevolution report on functional residue-residue interactions. A, Strong interaction between positions $x$ and $y$ induces coevolution. B, Negligibly weak interaction lets positions $x$ and $y$ evolve independently. In both cases an ancestral sequence (left) evolves along the same phylogenetic tree, whose bifurcations represent gene duplication or speciation events. Only two sequence positions, $x$ and $y$, are considered, the rest are indicated by dots. Encircled $x$ and $y$ characters mark substitution events at that position. As a result of substitutions, a sequence at the right (more recent) node of a branch may differ from the left (earlier) node at one or both positions. The process results in the set of contemporary sequences on the right, organized into a multiple alignment. Though substitutions occur stochastically in both cases, only in case $\mathbf{A}$ do they correlate temporally between the two positions. Since the protracted timescale of evolution precludes direct observation of substitution events, the challenge is to distinguish between the two cases $\mathbf{A}$ and $\mathbf{B}$ indirectly, using the alignment as the only available input data. 